\title{
Multimodality Imaging in the Evaluation of Cardiovascular Manifestations of Malignancy
}

\author{
Laura Jiménez-Juan, ${ }^{1}$ Jessica Leen, ${ }^{1}$ Rachel M. Wald, ${ }^{2}$ Elsie T. Nguyen, ${ }^{1}$ Andrew T. Yan, ${ }^{3}$ \\ Anish Kirpalani, ${ }^{3}$ Bernd J. Wintersperger, ${ }^{1,4}$ and Andrew M. Crean ${ }^{1,2,3,5}$ \\ ${ }^{1}$ Department of Medical Imaging, University Health Network, Toronto, ON, Canada M5G 2C4 \\ ${ }^{2}$ Division of Cardiology, Peter Munk Cardiac Centre, University Health Network, Toronto, ON, Canada M5G 2C4 \\ ${ }^{3}$ St. Michael's Hospital, Department of Medical Imaging, Toronto, ON, Canada M58 1WB \\ ${ }^{4}$ Department of Clinical Radiology, University of Munich Hospital, Ludwig Maximilians University, 80539 Munich, Germany \\ ${ }^{5}$ Department of Medical Imaging, Toronto General Hospital, 585 University Avenue, Toronto, ON, Canada M5G 2C4
}

Correspondence should be addressed to Andrew M. Crean, crean67@hotmail.com

Received 12 October 2010; Accepted 3 January 2011

Academic Editor: Syed Wamique Yusuf

Copyright ( $) 2011$ Laura Jiménez-Juan et al. This is an open access article distributed under the Creative Commons Attribution License, which permits unrestricted use, distribution, and reproduction in any medium, provided the original work is properly cited.

\begin{abstract}
Up to one third of the population will die as a direct result of cancer. Accurate and timely diagnosis of disease often requires multiple different approaches including the use of modern imaging techniques. Prompt recognition of adverse consequences of some anti-cancer therapies also requires a knowledge of the optimum imaging strategy for the problem at hand. The purpose of this article is to review not only some of the commoner cardiovascular manifestations of malignancy but also to discuss the strengths, weaknesses and appropriate use of cardiovascular imaging modalities.
\end{abstract}

\section{Introduction}

The heart is sometimes overlooked when considering the myriad systemic effects of cancer. However, cardiovascular manifestations of malignant disease (or its treatment) are not rare, are often significant, and have historically been diagnosed late in the natural history of diseaseor even remained undetected until postmortem. In the past, clinicians relied upon echocardiography to confirm cardiac involvement in malignant disease. However, contemporaneous noninvasive cardiac imaging with computed tomography (CT) and cardiovascular magnetic resonance (CMR) offers unparalleled opportunities for early diagnosis and surveillance in this patient population.

This paper will focus on the specific (and often complementary) roles of the four principal noninvasive imaging modalities (echocardiography, multidetector computed tomography (MDCT), cardiac magnetic resonance (CMR), and nuclear medicine) in patients with cancer $[1,2]$. Because there is considerable overlap between the techniques in regard to their clinical indications, our goal is to familiarize the clinician with the strengths and limitations of each modality to facilitate rational investigation for each specific clinical scenario.

\section{Noninvasive Imaging Modalities for Evaluation of Cardiac Masses and Malignant Cardiovascular Disease}

2.1. Echocardiography. Echocardiography remains the primary mode of initial investigation in suspected cardiovascular involvement by malignant disease [3]. This is by virtue not only of its availability, portability, and low cost but also because of its uniquely high temporal resolution, which offers a "real-time" assessment of cardiac structures that is currently unmatchable by CT or CMR at a comparable spatial resolution [4]. Fast-moving structures like the mitral or aortic valves can be accurately displayed over time [5]. Therefore, valvular involvement by disease, both malignant and infective (as a possible consequence of neutropenia), requires a very high temporal resolution in anemic, septic, 


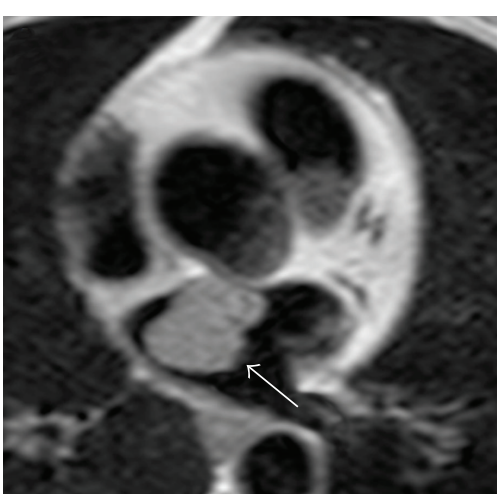

(a)

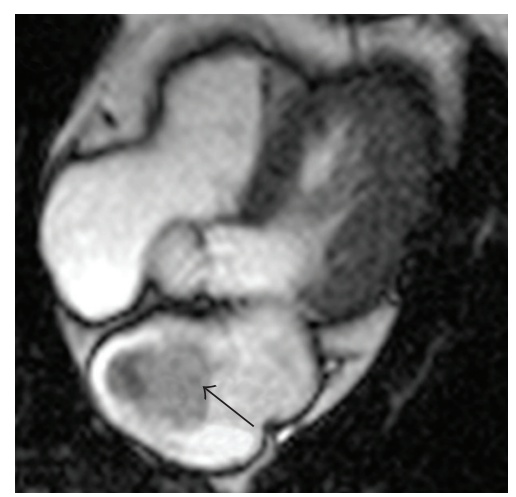

(c)

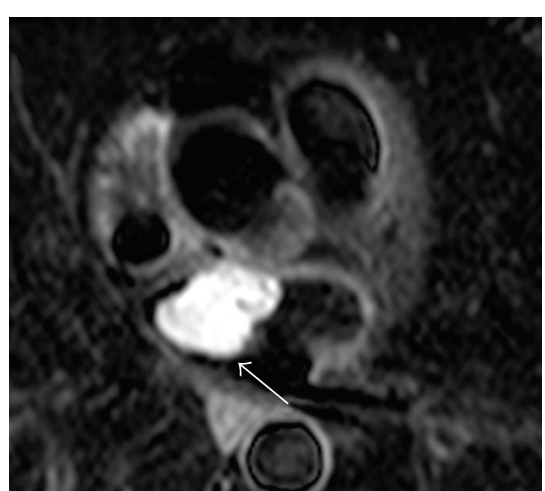

(b)

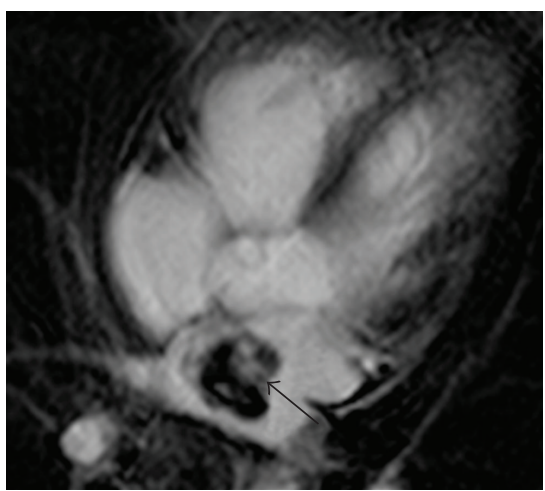

(d)

FIGURE 1: Left atrial myxoma. A lobulated mass is situated in the left atrium adjacent to the interatrial septum (a). On T2-weighted images, the mass appears uniformly high signal ((b), white arrow). Note that the mass is less well appreciated on bright blood cine imaging ((c), black arrow) and could be overlooked if it were smaller. Late gadolinium enhancement images (d) demonstrate patchy uptake of contrast within the mass (black arrow). The location and imaging characteristics are fairly typical for myxoma.

and tachycardic patients. Transesophageal echo (TEE) offers the advantage of a rapid and very high resolution survey of the valves and cardiac chambers in patients who may be too sick to undergo any other form of imaging [6].

A further advantage of echocardiography is its relative robustness in the face of sustained arrhythmia or frequent premature atrial or ventricular contractions. Image quality can be maintained in the face of significant rhythm disturbance unlike CT and CMR which usually "construct" an image based on an average of data derived from multiple sequential heart beats using a process known as "segmentation." Thus the varying cardiac cycle lengths introduced by arrhythmia cause considerable image perturbation frequently rendering the study nondiagnostic. Echocardiography is not subject to these constraints since multiple frames are acquired real-time in a single cardiac cycle, avoiding the need for segmentation [7].

Newer techniques in echocardiography have been less well studied in malignancy. Interrogation of myocardial strain using tissue Doppler or speckle tracking may provide earlier warning of chemotherapy-induced dysfunction than standard 2D echo techniques [8]. Three-dimensional echocardiography is in its infancy, and its clinical utility in the diagnostic evaluation of malignancy has not yet been well characterized [9].
2.2. Multidetector Computed Tomography (MDCT). CT is a versatile technique that has evolved rapidly in the last decade. Multidetector computed tomography (MDCT) was revolutionized by the invention of the 4 slice scanner in 2001 and has progressed through iterations that have involved $16,32,64,256$, and currently 320 detector rows. This has reduced the time required to scan an entire thorax from 40 seconds to a minimum of only 1 or 2 seconds. This is especially valuable for sick, intubated, or claustrophobic patients. The utility of MDCT has been further increased with the development of software for the scanner that permits cardiac gating. Images may be acquired at a relatively quiescent point towards end diastole, effectively "freezing" all cardiac motion. This, combined with the exquisite spatial resolution of MDCT, offers major advantages for assessment of the cardiac chambers, pericardium and surrounding vascular structures [10].

Not only does cardiac MDCT have high spatial resolution (0.5-0.65 $\mathrm{mm}$ in most cases) but this resolution is isotropic, that is, the lengths of the imaging element-the voxelare equal in $x, y$, and $z$ axes. This permits reconstruction of the raw data set (acquired in the axial plane) along any axis desired without any discernible loss of image quality. Interrogation of an abnormality in any plane desired makes MDCT an extremely powerful tool. This reformatting 
TABLE 1: Relative strengths and weaknesses of non-invasive imaging modalities.

\begin{tabular}{|c|c|c|c|c|}
\hline & ECHO & MDCT & CMR & RNA \\
\hline Availability & ++++ & +++ & + & ++ \\
\hline Temporal resolution & ++++ & ++ & +++ & + \\
\hline Spatial resolution & ++ & ++++ & +++ & + \\
\hline Contrast resolution & ++ & +++ & ++++ & + \\
\hline Cost & Low & Medium & High & Low \\
\hline Thoracic vessels & + & ++++ & ++++ & - \\
\hline Cardiac function & +++ & ++ & ++++ & +++ \\
\hline Speed of acquisition & +++ & ++++ & + & ++ \\
\hline Radiation & None & Moderate & None & Moderate \\
\hline Tissue characterization & + & ++ & +++ & - \\
\hline Suitability for sick or claustrophobic patients & ++++ & ++++ & $+1-$ & $+1-$ \\
\hline
\end{tabular}

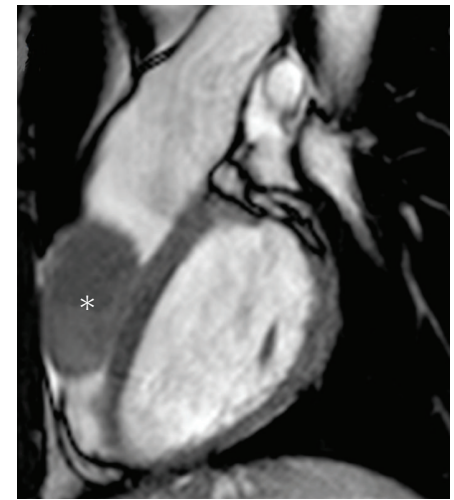

(a)

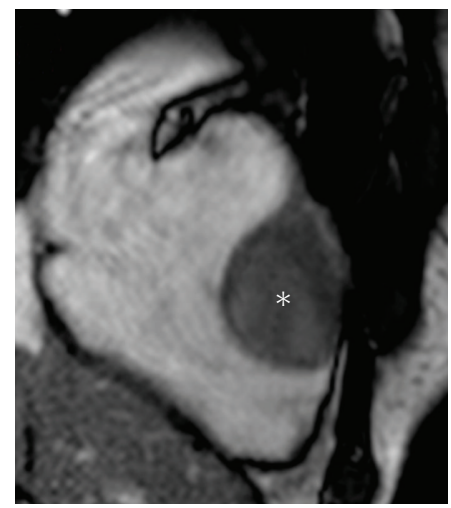

(d)

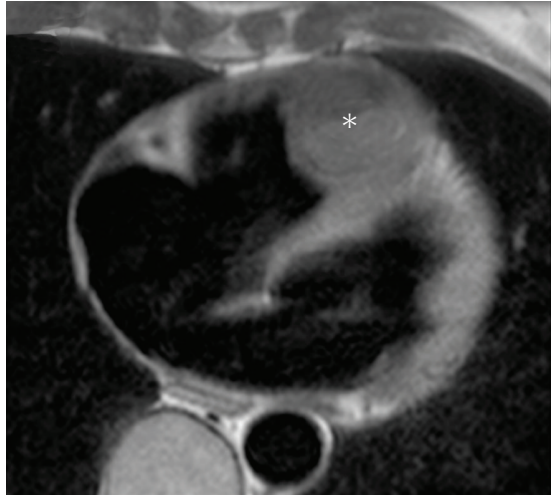

(b)

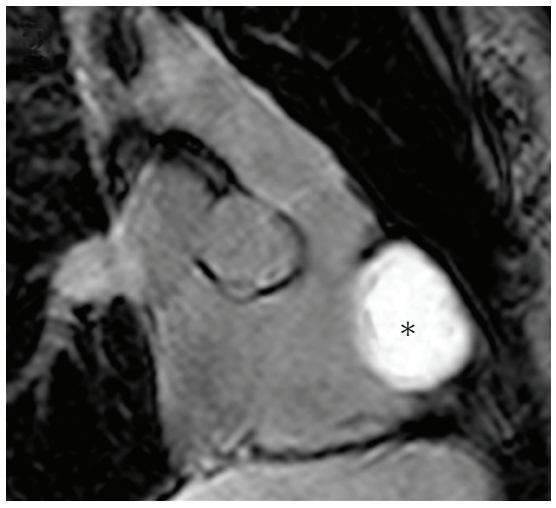

(e)

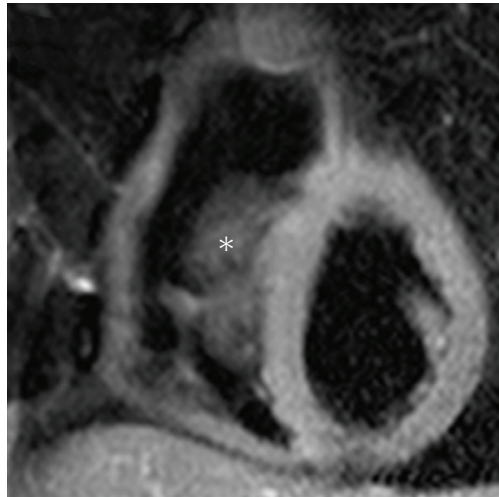

(c)

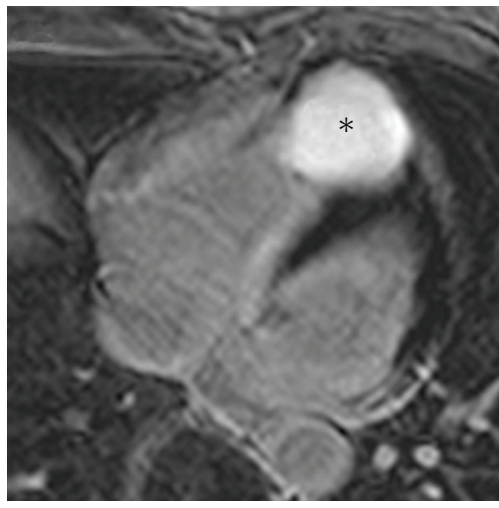

(f)

Figure 2: Fibroma. An ovoid mass partially obstructs the right ventricular outflow tract ((a)-(f), asterisk). The mass is of intermediate intensity on T1-weighted imaging ((a) and (b)) and low signal on T2-weighted imaging (c). This combination of findings would make malignancy relatively unlikely since many neoplasms have a relatively high water content and thus are high signal on T2 sequences. Dramatic contrast uptake is, however, evident on the postgadolinium images ((e) and (f)). Dense fibrous tumors may behave in this way, and the diagnosis of fibroma was later confirmed.

occurs after the patient has left, which allows flexibility in postprocessing workflow. This is an important distinguishing feature from the common bright-blood gradient echo or steady-state sequences used to evaluate cardiac anatomy and function in CMR, which are not isotropic and require accurate planning of cardiac planes prospectively.
Noncontrast MDCT is of limited value for soft tissue assessment other than lung parenchyma. It is, however, very sensitive for calcium and is the technique of choice to confirm pericardial calcification in the context of possible constriction. For assessment of the cardiac chambers and thoracic vasculature, administration of iodinated contrast 


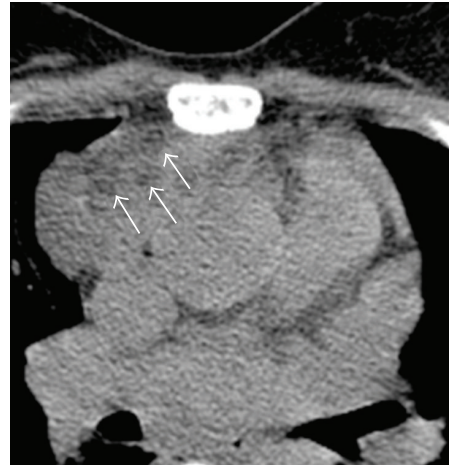

(a)

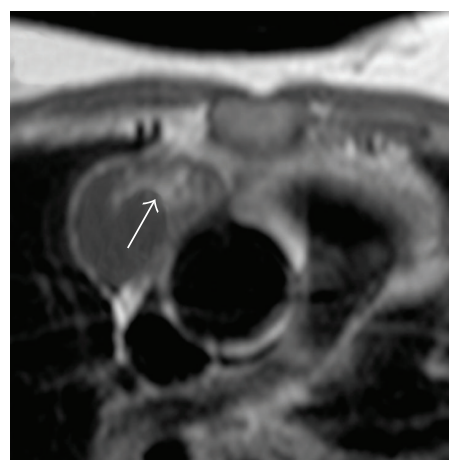

(d)

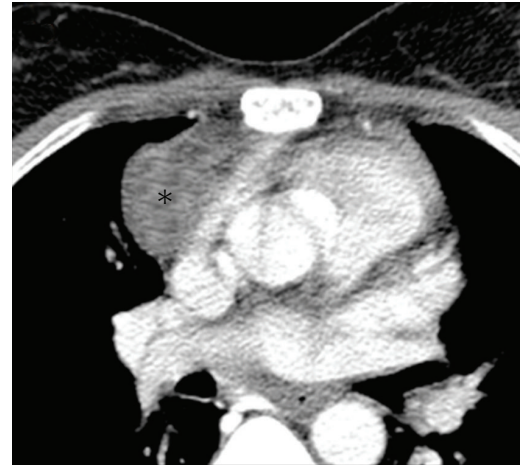

(b)

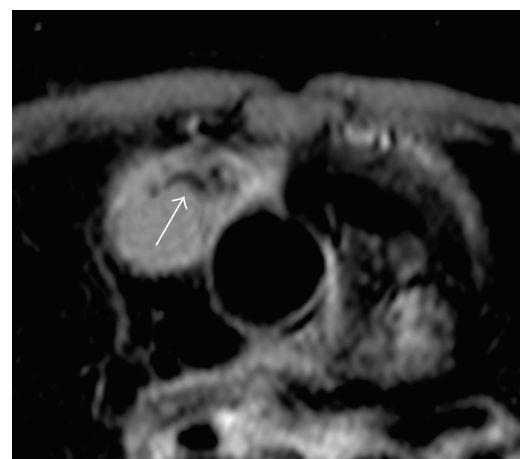

(e)

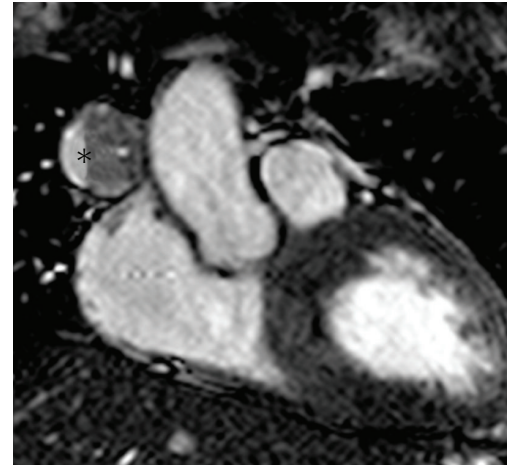

(c)

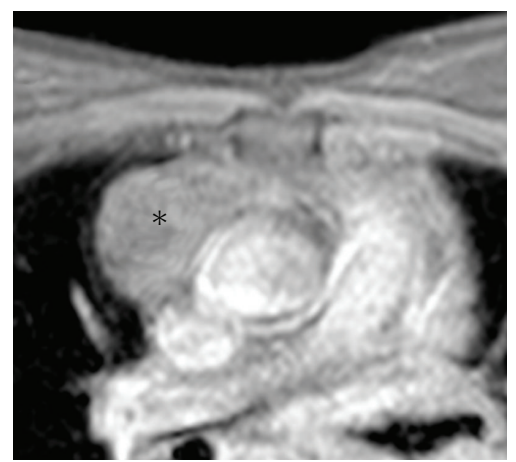

(f)

FIgURE 3: Teratoma. A well-circumscribed solid mass is present adjacent to the right atrial appendage ((b), (c), (f) asterisk). Foci of low attenuation are identified within it on noncontrast axial MDCT ((a), white arrows). Similar areas can be seen on the T1-weighted image ((d), white arrow) and show signal "dropout" with a fat-suppressed sequence ((e), white arrow). The presence of fat in a mixed density mediastinal lesion raised the possibility of teratoma, and this diagnosis was confirmed at pathology.

medium is required. This poses a problem in patients with chronic kidney disease, especially in the presence of diabetes. This may in some cases constitute a contraindication to the study because of the risk of contrast-induced nephropathy (CIN). Patients with GFR lower than threshold (often defined as $<30 \mathrm{~mL} / \mathrm{min} / \mathrm{kg}$ ) may receive contrast after careful consideration of risk/benefit ratio but the possibility of worsening nephropathy and potential dialysis has to be discussed [11, 12].

The principal disadvantage of MDCT is patient radiation exposure. However, this needs to be considered in the context of the patient's pre-existing malignancy and the overall likelihood of benefit from the scan [13]. Thoracic MDCT doses vary widely according to the scan parameters used. The mean effective dose can be anywhere between 5 and $25 \mathrm{mSv}$ for a single examination (the minimum and maximum doses quoted are roughly the same as for conventional coronary angiography and technetium MIBI nuclear study, resp.). Of particular concern is the radiation dose to the female breasts, lungs, and esophagus/thymus because these organs are sensitive to radiation-induced carcinogenesis [14].

2.3. Cardiovascular Magnetic Resonance (CMR). CMR is generally underutilized in the assessment of cardiovascular malignancy. This is primarily because of a lack of availability and expertise in this field outside of large centers.

CMR combines good spatial and acceptable (segmented) temporal resolution with unsurpassed soft tissue contrast resolution over a much wider dynamic range than MDCT. Not only is it excellent for anatomical assessment, it also provides hemodynamic information regarding flow velocity, biventricular volume and systolic function, and tissue characterization. The latter is a major strength; T2-weighted sequences may demonstrate that a mass has a high water content (raising suspicion of neoplasia in the appropriate context) or may, for example, demonstrate pericardial inflammation after radiotherapy as a cause for a patient's atypical chest pain. Administration of gadolinium-based contrast agents (GBCAs) can be combined with imaging sequences that are particularly sensitive to the uptake of gadolinium. The rate of uptake and pattern of enhancement may offer further insights into the underlying pathology and frequently enable a distinction between tumor and thrombus to be made with confidence [15]. Nonetheless CMR does not allow precise histopathological diagnosis in the majority of cases.

Measurement of LV volumes and function is highly reproducible by CMR, much more so than for any other modality [16]. Screening for small decrements in ventricular 


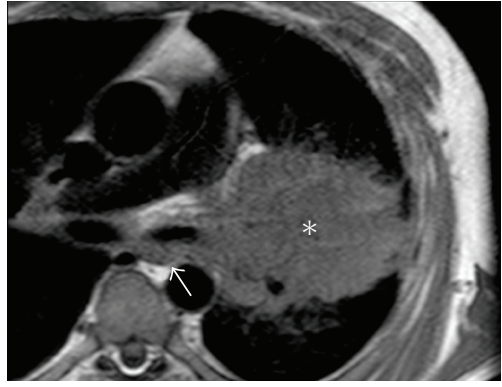

(a)

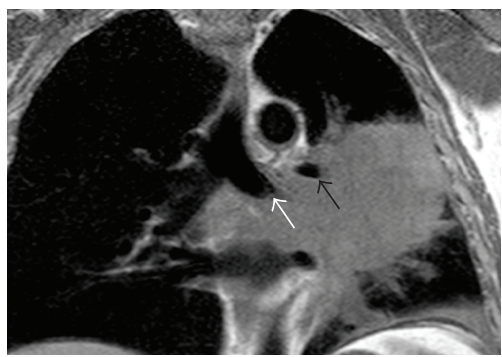

(c)

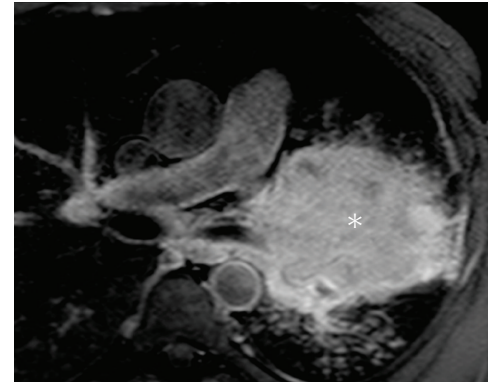

(b)

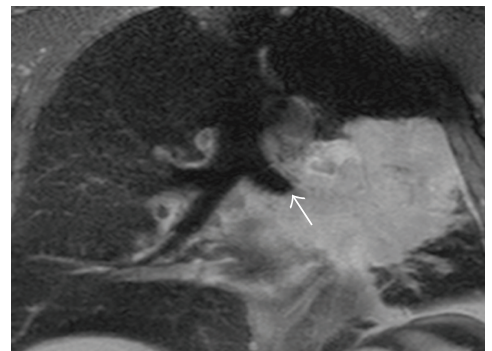

(d)

FIgURE 4: Bronchogenic carcinoma. A large mass (asterisk) is shown in axial ((a) and (b)) and coronal ((c) and (d)) planes. On T1-weighted images the mass demonstrates significant near-uniform enhancement after gadolinium contrast ((a) and (c) before contrast; (b) and (d) after contrast). The mass infiltrates posterior to the carina ((a), white arrow) and occludes the left main bronchus ((c) and (d), white arrow). The left main pulmonary artery is severely attenuated ((c), black arrow).

TABLE 2: Chemotherapeutic agents associated with left ventricular dysfunction.

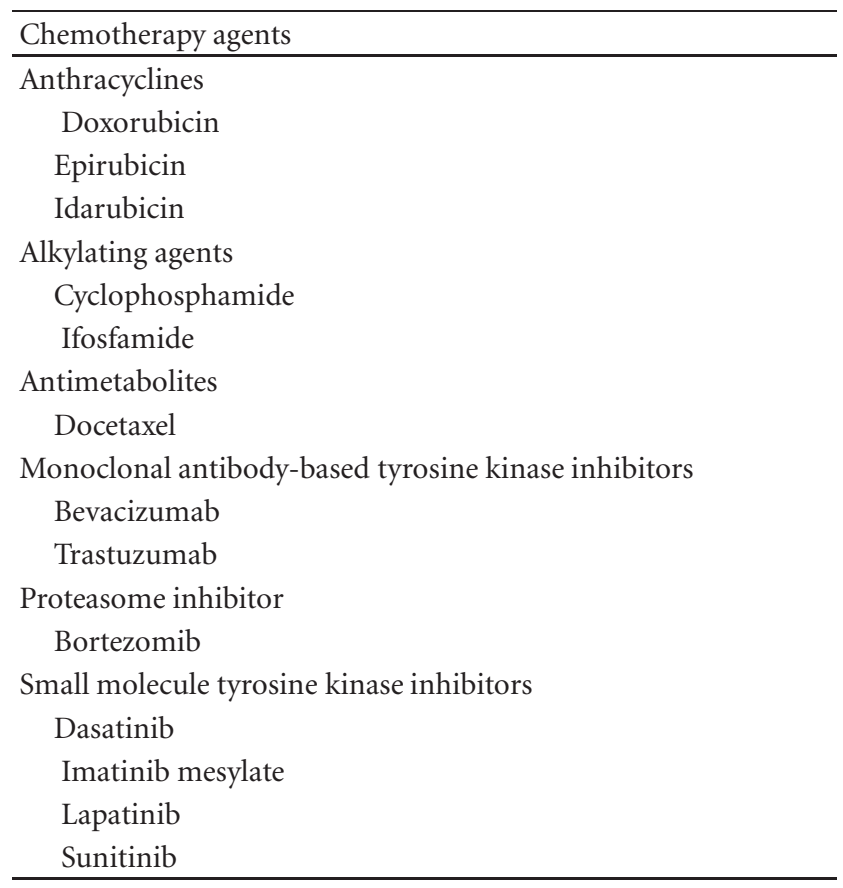

function in patients receiving cardiotoxic chemotherapy is a function that has been historically performed by radionuclide angiography. In the future it seems probable that CMR will take on at least a portion of this work perhaps targeting the younger patients who have most to gain from sequential followup with a technique that involves no ionizing radiation.

Angiographic delineation of the major vascular structures in the chest is readily achieved by CMR using both static "snapshot" imaging and time-resolved magnetic resonance angiography which delineates the passage of contrast through the vascular tree providing differential enhancement of arterial and venous structures. This may be useful in establishing degree of obstruction in, for example, SVC syndrome as well as facilitating interventional planning.

A certain amount of subjective information may also be gained about relative lung perfusion in cases of unilateral tumor involvement of the pulmonary arteries or veins, with delayed parenchymal blush often occurring on the obstructed side. Formal measurements of relative flow may be made using phase velocity mapping which is a flow sensitive technique akin to echo Doppler. The ability to interrogate a vessel in any plane renders CMR far more flexible than its sister modality.

CMR has both limitations and definite exclusions. Amongst the former, a number lack availability and expertise both to perform and interpret the studies. CMR is also rarely a rapid method of gathering data and so is poorly tolerated by the claustrophobic and very unwell for whom the challenge of remaining still and breath holding repetitively on command is often insurmountable. Symptomatic claustrophobia is very common occurring in up to $25 \%$ of patients although only $5-6 \%$ of all patients fail to complete an exam for this reason [17]. 


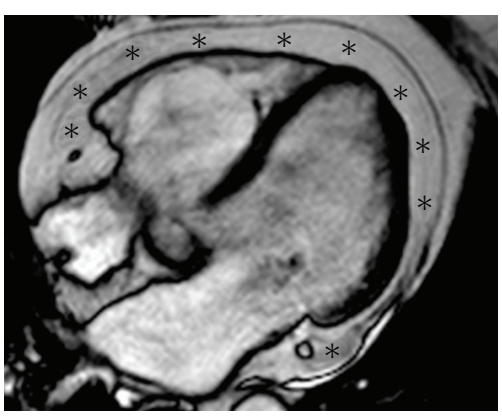

(a)

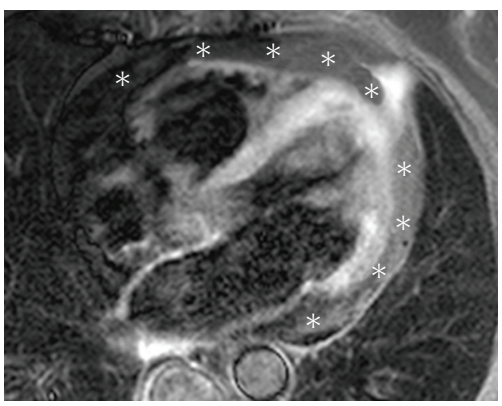

(c)

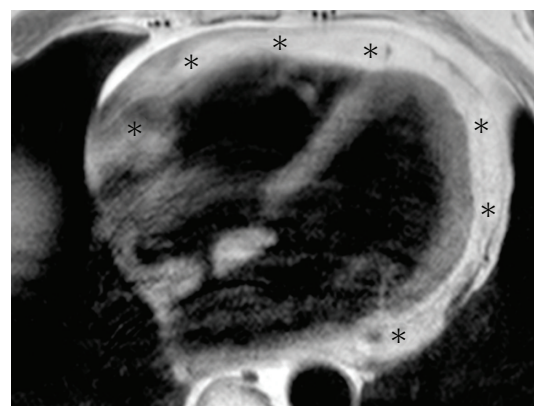

(b)

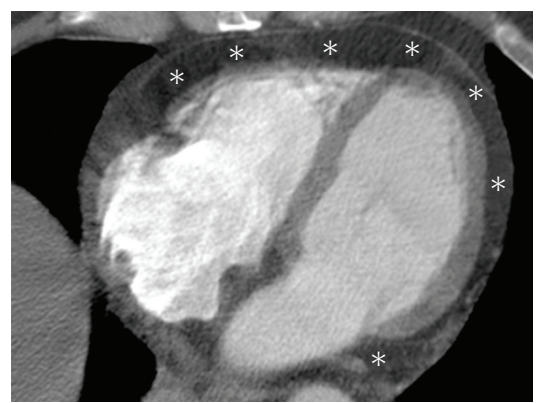

(d)

Figure 5: Epicardial fat. Echocardiography raised concerns about excessive soft tissue anterior to the right ventricle in this patient on long-term steroids. Bright blood cine (a) and T1-weighted images (b) show circumferential high signal around the heart (asterisks). The appearances suggest an unusual amount of pericardial fat, and this was confirmed on fat-suppressed CMR (c) where there is evidence of signal drop out from the fatty region (asterisks - compare with (b)). MDCT is also very sensitive for fat which it displays as low-density regions of negative Hounsfield attenuation ((d), asterisks). Excessive mediastinal lipomatosis is not uncommon in patients taking steroid preparations.

Critically ill patients may be unable to enter the scan room if attached to standard infusion pumps because of possible magnetic interference. Similarly the presence of a pacemaker or defibrillator currently remains a near-absolute contraindication to CMR in most institutions worldwide.

Pregnancy is not an absolute contraindication of itself; attempts are generally made to keep the radiofrequency power deposition levels as low as possible primarily because of theoretical concerns about heating of the fetus. Similarly gadolinium contrast is generally not given although there are no data to suggest harm to the fetus at standard clinical doses.

In many of the situations where CMR is difficult or contraindicated, the patient may be equally well served by a timely MDCT study plus echo where function or flow data is vital.

2.4. Nuclear Medicine: Radionuclide Angiography (RNA). The main goal of RNA is the visualization of the cardiac chambers and great vessels. This requires the temporary labeling of the blood with a suitable radioactive tracer. It provides an accurate evaluation of the structure and function of the heart, including ejection fraction, regional wall motion variability, and diastolic function [18].

RNA is widely used for the assessment of the cardiac function after chemotherapy, principally because of its ready availability [19]. However, although its reproducibility is very high, the main disadvantages of RNA are its limited temporal resolution and the use of ionizing radiation (approx. 3$6 \mathrm{mSv}$ ), which makes CMR a more attractive and highly reproducible method of evaluating cardiac function without a radiation penalty $[20]$.

The relative merits and demerits of each of the 4 principal modalities discussed above are summarized in Table 1.

\section{Multi-Modality Imaging in Specific Clinical Scenarios}

3.1. Characterisation of Cardiac Tumors. Cardiac tumors may be categorised as either primary or secondary. Primary tumors are rare, ranging from $0.001 \%$ to $0.030 \%$ [21] and three quarters of these tumors are benign. The most common primary tumor is myxoma (Figure 1) (about 50\%); the remainder includes lipoma, papillary fibroelastoma, cavernous hemangioma, fibroma (Figure 2), rhabdomyoma, paraganglioma, mediastinal teratoma (Figure 3), and lymphangioma. Of the malignant primary tumours, about $95 \%$ are sarcomas and 5\% are primary lymphomas and primary cardiac carcinoid.

Secondary tumors, however, are much more common than primary tumors, with an incidence of metastasis to heart of $10 \%[22,23]$. The most frequent underlying malignant origin is the lung (Figure 4). Secondary involvement may be the result of direct invasion, lymphatic or 


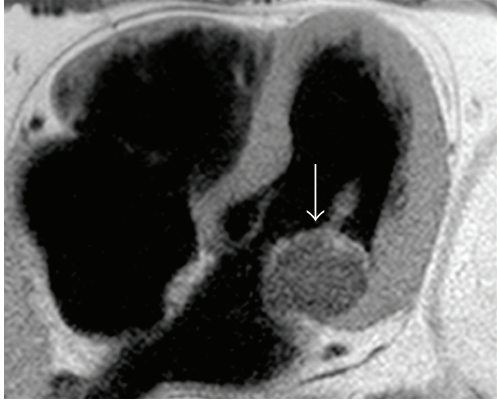

(a)

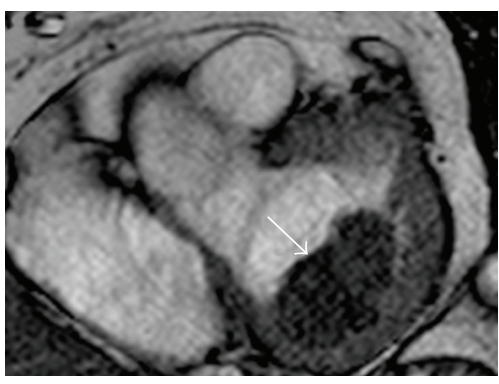

(c)

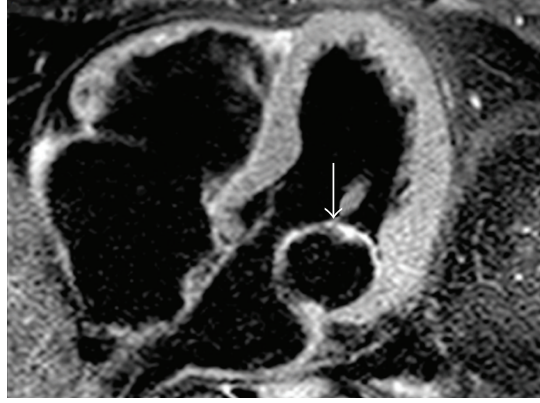

(b)

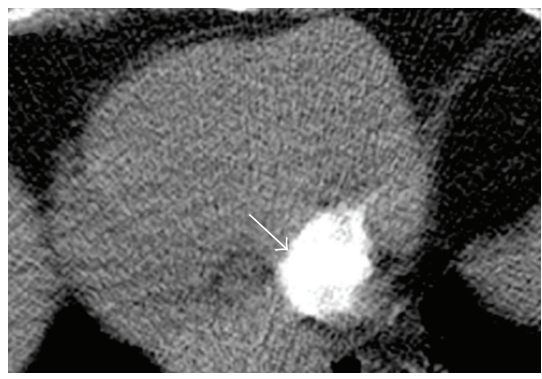

(d)

Figure 6: Mitral annular calcification (MAC). A classic "pseudotumour" MAC is seen in older patients, often with a history of diabetes or hypertension. It presents as focal mass-like lesions most frequently adjacent to the lateral mitral annulus ((a) and (b), white arrow). In this example the signal characteristics on CMR are typically being low signal on T1-weighted images (a) and T2-weighted images (b). Its relation to the posterior mitral annulus is well appreciated on the short axis bright-blood cine view ((c), white arrow). Noncontrast MDCT excludes any residual diagnosis about the diagnosis — the mass is of very high attenuation consistent with calcium ((d), white arrow).

hematogenous spread. In rare cases, direct transvascular extension may occur (e.g., renal cell carcinoma extending into the right heart via the inferior vena cava). The detection of a cardiac mass should therefore always lead to a screen for primary malignancy elsewhere in the body.

There is a group of nonneoplastic conditions of the heart and pericardium that can mimic cardiac tumors. These include pericardial cysts, lymphadenopathy, lipomatous hypertrophy of the atrial septum, pericardial fat (Figure 5), caseous calcification of mitral valve [24], coronary artery aneurysm, and thrombus. Despite the lack of malignant potential of these lesions, they can be associated with considerable morbidity and even mortality due to inappropriate treatment. For this reason, their recognition and differentiation from malignant tumors is crucial [25]. The most frequent pseudotumor is thrombus, which is discussed separately below.

For diagnosis of cardiac tumors, TTE and TEE are widely used as they can localize the tumor and give information about size, shape, attachment, and mobility. Contrast echocardiography is also used for assessment of intracardiac masses [26]. However, for complex tumors and large or infiltrating tumors, the combination of echocardiography with either MRI or MDCT provides better definition and more precise preoperative planning [27].

Tumor breach of tissue planes is often depicted with greater certainty by MDCT than CMR due to better spatial resolution. Detection of small amounts of tumor may be better by CMR because of its superior contrast resolution for soft tissue. The presence of calcium, however, is recognised with greater ease using MDCT than CMR which can be helpful when assessing mitral valve masses (Figure 6).

3.2. Thrombus. Thrombus is the most common intracardiac mass involving the left ventricle or left atrium [28]. Its identification and proper characterization is very important so that anticoagulant therapy is not delayed (Figure 7). Although TTE can detect the presence of thrombus and is usually the primary screening tool, it has limited sensitivity for this purpose [29]. Laminated mural thrombi may be difficult to distinguish from myocardium and slow blood flow within aneurysms. Late gadolinium enhancement CMR is very sensitive for the presence of thrombus [30]. Several distinguishing CT imaging features have been described to differentiate thrombus from other tumors including size, origin, shape, and prolapse [31]. However, the accuracy of MDCT in this regard has not been established in any large studies.

3.3. Pericardial Disease. Pericardial disease related to cancer encompasses both the direct effects of tumor invasion as well as the indirect consequences of thoracic surgery and/or radiotherapy.

The initial evaluation for pericardial effusion is usually by transthoracic echo. Echocardiography is useful in evaluating the physiological changes in pericardial diseases [32]. Malignant pericardial effusion is not uncommon, and TTE is generally the most sensitive method for detecting 


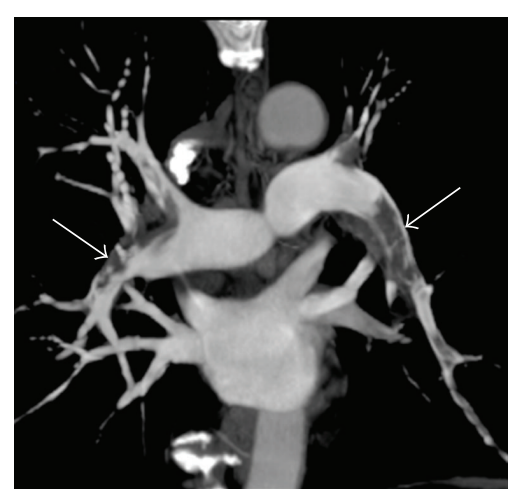

(a)

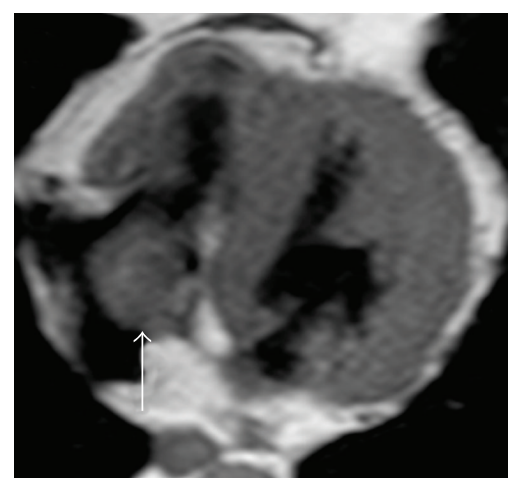

(d)

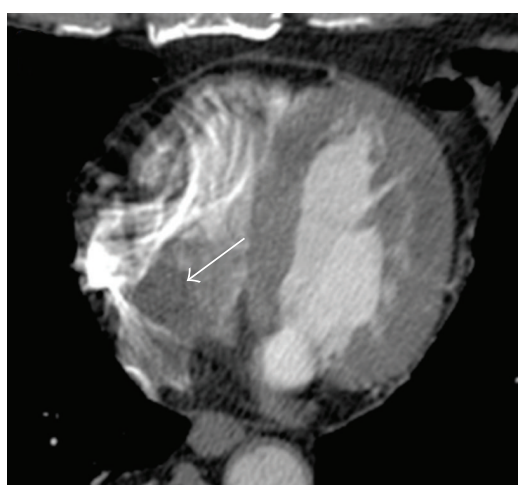

(b)

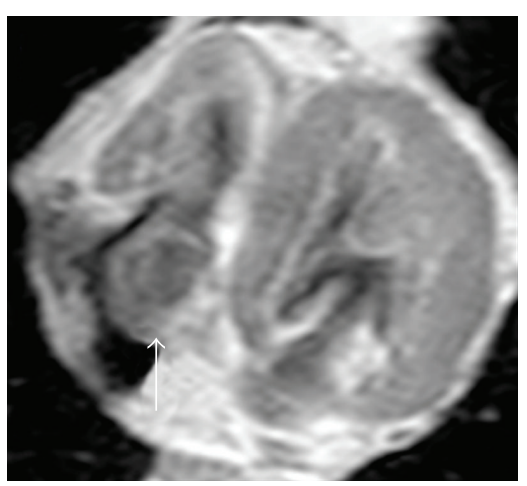

(e)

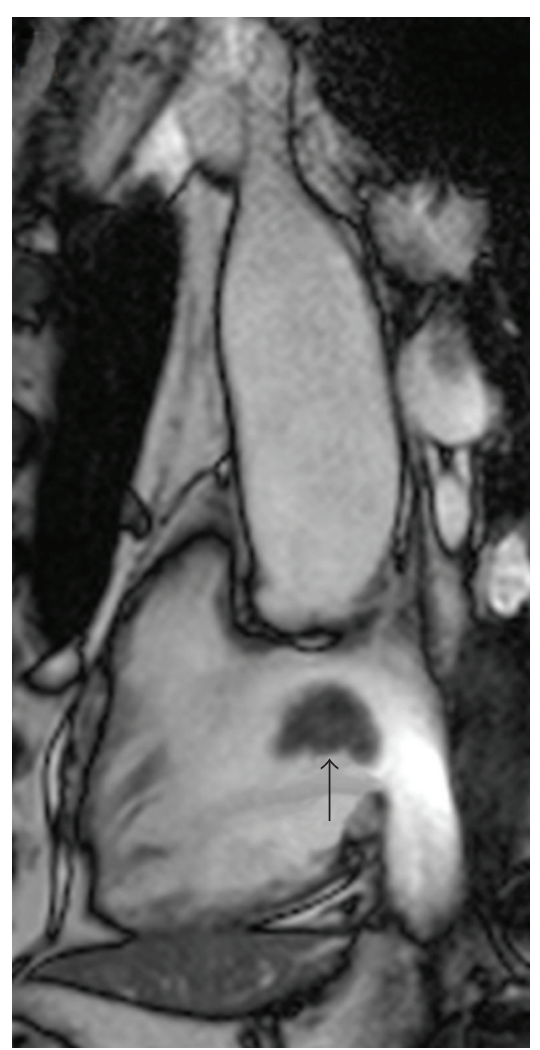

(c)

Figure 7: Thrombus. A coronal maximum intensity projection image from a contrast MDCT study demonstrates multiple pulmonary emboli ((a), white arrows). On the same study the possibility of a right atrial mass was raised ((b), white arrow), although it was uncertain whether this was real or simply an appearance due to incomplete mixing of opacified SVC blood with unopacified IVC blood. Cine CMR demonstrates that the mass is clearly real ((c), black arrow). Further characterization by CMR demonstrates that the mass is low signal on T1 weighted images $((\mathrm{d})$, white arrow) and remains low signal following gadolinium administration ((e), white arrow). The appearances and clinical presentation were entirely consistent with right atrial thrombus. The mass disappeared with subsequent anticoagulation.

both incipient and frank tamponade (Figure 8). However, it is limited in the assessment of pericardial extension, pericardial thickening, and inflammation, whereas CMR and MDCT excel in this regard. MDCT is the best technique for depiction of pericardial calcification. In addition, the composition of pericardial collections (simple or complex) may be inferred from measurements of Hounsfield density. This may guide subsequent management since complex collections are unlikely to respond well to percutaneous drainage and often require surgical intervention.

CMR is better at differentiating small pericardial effusions from pericardial thickening and provides a functional and structural assessment of the pericardium. CMR can also evaluate physiological consequences of pericardial constriction. Specific imaging techniques for pericardial constriction include cine-tagged imaging which can demonstrate tethering of the myocardium during systole. Real-time cine imaging may be used to demonstrate septal bounce seen in constriction [33].

CMR may show pericardial inflammation (e.g., acutely after radiotherapy) with T2-weighted or late gadolinium enhancement imaging.

\section{Cardiovascular Complications of Cancer Therapy}

The therapeutic options for patients with cancer include complex combinations of medications, radiation therapy, and surgery. Many of these treatments have important potential adverse cardiovascular effects. Indeed, cardiovascular events represent the second most common cause of mortality after cancer recurrence [34]. Although there is no unified consensus for the assessment and monitoring of the cardiovascular complications of cancer treatment, noninvasive imaging is increasingly used [35].

4.1. Radiation. Mediastinal irradiation is not uncommon for solid and lymphomatous malignancies involving the chest [36]. The potential adverse effects include arteritis, pericarditis, cardiomyopathy, valvular disease, and conduction anomalies.

Radiation-induced valvular disease is limited, affecting approximately $6-15 \%$ of patients exposed to mediastinal radiation [37]. Valvular regurgitation is more common [38]. The typical findings include valvular thickening, fibrosis, and 


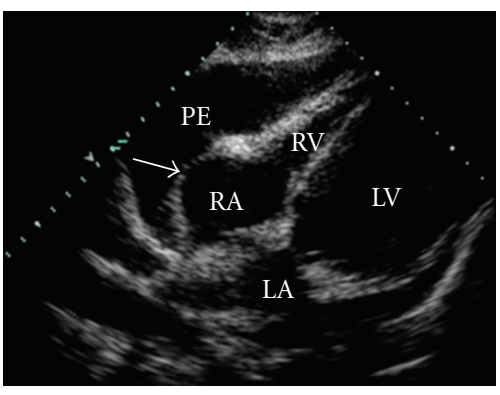

(a)

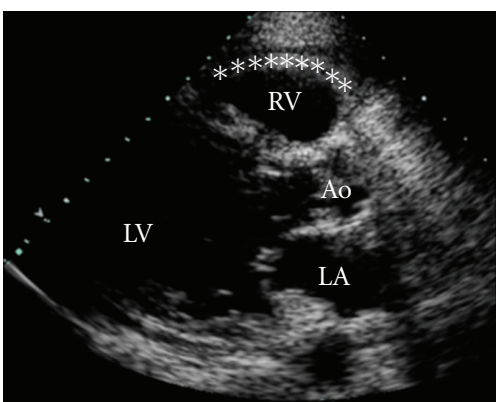

(c)

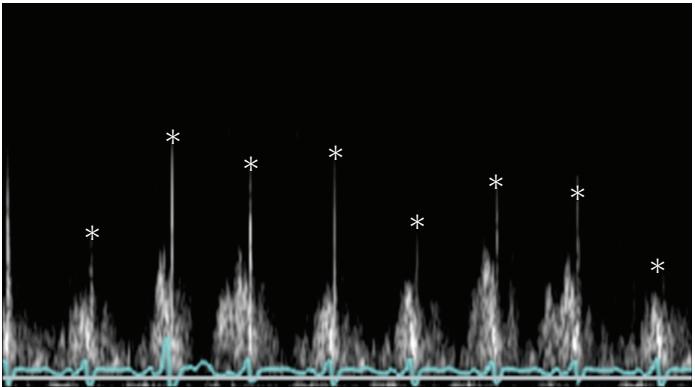

(e)

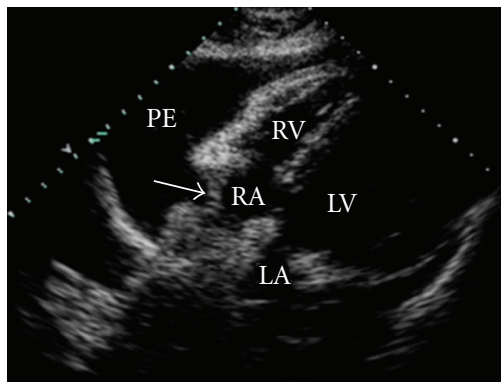

(b)

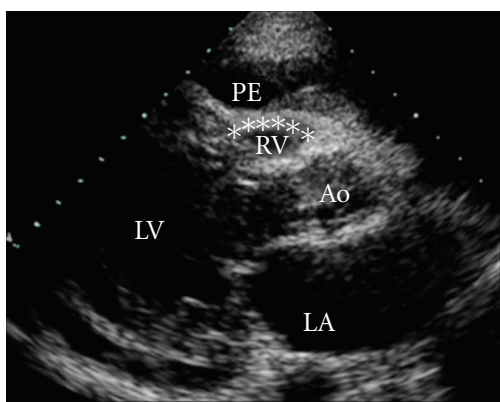

(d)

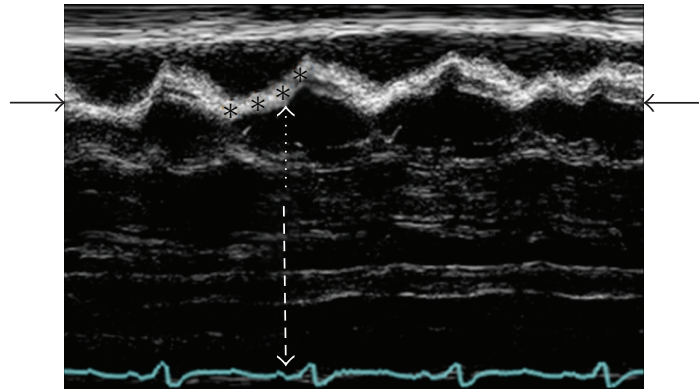

(f)

Figure 8: Echo signs of tamponade. Diastolic inversion of the right atrial (RA) free wall is an early sign of raised intrapericardial pressure ((a) and (b)), white arrow-note collapse/inversion of the RA free wall in early diastole (b) as the intrapericardial pressure exceeds the RA pressure. Right ventricular diastolic inversion is a later but more specific sign of tamponade ((c) and (d)) asterisks outline the RV free wallthe RV shows diastolic collapse (d) due to the adjacent effusion (PE). Note also the relative enlargement of the left ventricle (LV) in this case; a result of chemotherapy-related cardiomyopathy. Doppler techniques are often very helpful in establishing the diagnosis of tamponadetranstricuspid flow variability of greater than $33 \%$ on a beat-to-beat basis ((e), asterisks represent peak transtricuspid flow for each recorded beat) is suggestive of tamponade. M-mode Doppler has a very high sampling (frame) rate compared to standard B-mode Doppler and is therefore useful for timing the motion of the RV free wall with respect to the cardiac cycle ((f), horizontal white arrows indicate the RV free wall; as would be expected this moves in normally during systole but is slow to move outwards in early-to-mid diastole (black asterisks) —in fact full outward excursion only occurs late in diastole (dotted short arrow) aided by the filling from atrial contraction (dotted long arrow indicates $P$ wave on ECG, i.e., atrial systole)).

calcifications. TEE is the best imaging modality to evaluate valvular function.

Pericardial disease induced by radiation is typically a long-term complication and may develop years after treatment, manifesting as silent pericardial effusions or with constrictive pericarditis, with or without pericardial fluid. Echo together with CMR may both be required to support a clinical diagnosis of constriction. Cardiomyopathy after radiation is predominantly due to diastolic dysfunction with a restrictive pattern - these features may be most evident on echo which is best suited to evaluate abnormalities of diastolic function. This is primarily because of its very high temporal resolution which permits beat-to-beat (real-time) measurement of the transmitral flow abnormalities which characterize diastolic impairment.

4.2. Chemotherapy. Many chemotherapy drugs have the potential to cause cardiotoxicity. The chemotherapy agents associated with LV dysfunction are shown in Table 2. Different expert working groups have developed practice guidelines for cardiac monitoring requirements in patients 
undergoing chemotherapy. For example, the recommendations of the Canadian Trastuzumab working group include LVEF assessment before trastuzumab treatment is started and repeated every 3 months until completion of therapy. Several authors have also described development of myocardial fibrosis in conjunction with certain chemotherapeutic agents demonstrated by late gadolinium enhancement on CMR [39].

4.3. Others. The use of devices for chemotherapy, such as indwelling venous catheters, predisposes to thrombus formation typically at the tip of the catheter. This is often well depicted by CMR. MDCT can be confusing since incomplete mixing in the right atrium during the first pass of contrast often simulates filling defects-definite CT confirmation of a real mass is best achieved by performing a delayed acquisition 1-2 minutes after contrast injection.

\section{Conclusion}

The extent of potential cardiovascular involvement by malignancy is broad. Four main non-invasive imaging methods are available for evaluation of suspected cardiovascular complications of malignancy. They are useful for the exclusion of other diseases and to guide proper management of these patients. Non-invasive imagers are well educated in the strengths and weaknesses of the various imaging techniques; optimum use of this expensive technology is most efficient when proper dialogue occurs between cancer and imaging specialists.

\section{References}

[1] G. J. Klein and J. P. Thirion, "Cardiovascular imaging to quantify the evolution of cardiac diseases in clinical development," Biomarkers, vol. 10, supplement 1, pp. S1-S9, 2005.

[2] S. V. Del Castillo, R. Aguilar Torres, and J. C. Paré Bardera, "Update on cardiac imaging techniques: echocardiography, cardiac magnetic resonance, and multidetector computed tomography," Revista Espanola de Cardiologia, vol. 62, supplement 1, pp. 129-150, 2009.

[3] P. S. Douglas, B. Khandheria, R. F. Stainback et al., "ACCF/ASE/ACEP/ASNC/SCAI/SCCT/SCMR 2007 appropriateness criteria for transthoracic and transesophageal echocardiography," Journal of the American College of Cardiology, vol. 50, no. 2, pp. 187-204, 2007.

[4] G. Rao, N. Sajnani, L. L. Kusnetzky, and M. L. Main, "Appropriate use of transthoracic echocardiography," American Journal of Cardiology, vol. 105, no. 11, pp. 1640-1642, 2010.

[5] R. O. Bonow, B. A. Carabello, K. Chatterjee et al., "ACC/AHA 2006 guidelines for the management of patients with valvular heart disease," Journal of the American College of Cardiology, vol. 48, no. 3, pp. e1-e148, 2006.

[6] E. A. Tee and P. M. Shah, "Transesophageal echocardiography," Journal of Intensive Care Medicine, vol. 7, no. 3, pp. 113-126, 1992.

[7] J. D. Thomas and Z. B. Popović, "Assessment of left ventricular function by cardiac ultrasound," Journal of the American College of Cardiology, vol. 48, no. 10, pp. 2012-2025, 2006.
[8] J. L. Hare, J. K. Brown, R. Leano, C. Jenkins, N. Woodward, and T. H. Marwick, "Use of myocardial deformation imaging to detect preclinical myocardial dysfunction before conventional measures in patients undergoing breast cancer treatment with trastuzumab," The American Heart Journal, vol. 158, no. 2, pp. 294-301, 2009.

[9] T. H. Marwick, "Echocardiography in the era of multimodality imaging," Heart Lung and Circulation, vol. 19, no. 3, pp. 175$184,2010$.

[10] U. J. Schoepf, C. R. Becket, L. K. Hofmann, and E. K. Yucel, "Multidetector-row CT of the heart," Radiologic Clinics of North America, vol. 41, no. 3, pp. 491-505, 2003.

[11] P. A. McCullough, A. Adam, C. R. Becker et al., "Risk prediction of contrast-induced nephropathy," American Journal of Cardiology, vol. 98, no. 6A, pp. 27K-36K, 2006.

[12] N. Lameire, A. Adam, C. R. Becker et al., "Baseline renal function screening," American Journal of Cardiology, vol. 98, no. 6A, pp. 21K-26K, 2006.

[13] G. Bastarrika, Y. S. Lee, W. Huda, B. Ruzsics, P. Costello, and U. J. Schoepf, "CT of coronary artery disease," Radiology, vol. 253, no. 2, pp. 317-338, 2009.

[14] E. L. Nickoloff and P. O. Alderson, "A comparative study of thoracic radiation doses from 64-slice cardiac CT," British Journal of Radiology, vol. 80, no. 955, pp. 537-544, 2007.

[15] D. H. O'Donnell, S. Abbara, V. Chaithiraphan et al., "Cardiac tumors: optimal cardiac MR sequences and spectrum of imaging appearances," American Journal of Roentgenology, vol. 193, no. 2, pp. 377-387, 2009.

[16] R. Hoffmann, S. Von Bardeleben, F. ten Cate et al., "Assessment of systolic left ventricular function: a multi-centre comparison of cineventriculography, cardiac magnetic resonance imaging, unenhanced and contrast-enhanced echocardiography," European Heart Journal, vol. 26, no. 6, pp. 607-616, 2005.

[17] J. M. Francis and D. J. Pennell, "Treatment of claustrophobia for cardiovascular magnetic resonance: use and effectiveness of mild sedation," Journal of Cardiovascular Magnetic Resonance, vol. 2, no. 2, pp. 139-141, 2000.

[18] S. C. Port and F. J. Wackers, "Clinical application of radionuclide angiography," Journal of Nuclear Cardiology, vol. 2, no. 6, pp. 551-558, 1995.

[19] M. J. Aiken, V. Suhag, C. A. Garcia et al., "Doxorubicininduced cardiac toxicity and cardiac rest gated blood pool imaging," Clinical Nuclear Medicine, vol. 34, no. 11, pp. 762767, 2009.

[20] F. J. Klocke, M. G. Baird, B. H. Lorell et al., "ACC/AHA/ASNC guidelines for the clinical use of cardiac radionuclide imaging - executive summary," Journal of the American College of Cardiology, vol. 42, no. 7, pp. 1318-1333, 2003.

[21] J. Butany, V. Nair, A. Naseemuddin, G. M. Nair, C. Catton, and T. Yau, "Cardiac tumours: diagnosis and management," Lancet Oncology, vol. 6, no. 4, pp. 219-228, 2005.

[22] C. Gaudio, G. Tanzilli, P. Mazzarotto et al., "Comparison of left ventricular ejection fraction by magnetic resonance imaging and radionuclide ventriculography in idiopathic dilated cardiomyopathy," American Journal of Cardiology, vol. 67, no. 5, pp. 411-415, 1991.

[23] J. O. Wee, J. D. Sepic, T. Mihaljevic, and L. H. Cohn, "Metastatic carcinoid tumor of the heart," Annals of Thoracic Surgery, vol. 76, no. 5, pp. 1721-1722, 2003.

[24] C. Chiles, P. K. Woodard, F. R. Gutierrez, and K. M. Link, "Metastatic involvement of the heart and pericardium: CT and MR imaging," Radiographics, vol. 21, no. 2, pp. 439-449, 2001.

[25] D. V. Miller and H. D. Tazelaar, "Cardiovascular pseudoneoplasms," Archives of Pathology and Laboratory Medicine, vol. 134, no. 3, pp. 362-368, 2010. 
[26] N. Mansencal, L. Revault-d'Allonnes, J. P. Pelage, J. C. Farcot, P. Lacombe, and O. Dubourg, "Usefulness of contrast echocardiography for assessment of intracardiac masses," Archives of Cardiovascular Diseases, vol. 102, no. 3, pp. 177-183, 2009.

[27] D. Trifunovic, B. Vujisic-Tesic, M. Vuckovic et al., "Multimodality imaging in the assessment of cardiac lymphoma presented as new-onset atrial fibrillation," Echocardiography, vol. 27, no. 3, pp. 332-336, 2010.

[28] B. Higgings and A. De Ross, MRI and CT of the Cardiovascular System, Lippincott Williams \& Wilkins, Philadelphia, Pa, USA, 2nd edition, 2005.

[29] J. Barkhausen, P. Hunold, H. Eggebrecht et al., "Detection and characterization of intracardiac thrombi on MR imaging," American Journal of Roentgenology, vol. 179, no. 6, pp. 15391544, 2002.

[30] M. B. Srichai, C. Junor, L. L. Rodriguez et al., "Clinical, imaging, and pathological characteristics of left ventricular thrombus: a comparison of contrast-enhanced magnetic resonance imaging, transthoracic echocardiography, and transesophageal echocardiography with surgical or pathological validation," American Heart Journal, vol. 152, no. 1, pp. 75-84, 2006.

[31] S. Tatli and M. J. Lipton, "CT for intracardiac thrombi and tumors," International Journal of Cardiovascular Imaging, vol. 21, no. 1, pp. 115-131, 2005.

[32] P. A. N. Chandraratna, "Echocardiography and doppler ultrasound in the evaluation of pericardial disease," Circulation, vol. 84, supplement 3, pp. I303-I310, 1991.

[33] R. Rienmüller, R. Gröll, and M. J. Lipton, "CT and MR imaging of pericardial disease," Radiologic Clinics of North America, vol. 42, no. 3, pp. 587-601, 2004.

[34] J. R. Carver, C. L. Shapiro, A. Ng et al., "American society of clinical oncology clinical evidence review on the ongoing care of adult cancer survivors: cardiac and pulmonary late effects," Journal of Clinical Oncology, vol. 25, no. 25, pp. 3991-4008, 2007.

[35] W. O. Ntim and W. G. Hundley, "Imaging surveillance for cardiovascular complications of cancer therapy," Journal of the American College of Cardiology, vol. 55, no. 2, pp. 171-172, 2010.

[36] W. Machann, M. Beer, M. Breunig et al., "Cardiac magnetic resonance imaging findings in 20-year survivors of mediastinal radiotherapy for hodgkin's disease," International Journal of Radiation Oncology, Biology, Physics, vol. 79, no. 4, pp. 1117$1123,2011$.

[37] M. C. Hull, C. G. Morris, C. J. Pepine, and N. P. Mendenhall, "Valvular dysfunction and carotid, subclavian, and coronary artery disease in survivors of hodgkin lymphoma treated with radiation therapy," Journal of the American Medical Association, vol. 290, no. 21, pp. 2831-2837, 2003.

[38] M. Malanca, C. Cimadevilla, E. Brochet, B. Iung, A. Vahanian, and D. Messika-Zeitoun, "Radiotherapy-induced mitral stenosis: a three-dimensional perspective," Journal of the American Society of Echocardiography, vol. 23, no. 1, pp. 108.e1-108.e2, 2010.

[39] R. D. Perel, R. E. Slaughter, and W. E. Strugnell, "Subendocardial late gadolinium enhancement in two patients with anthracycline cardiotoxicity following treatment for Ewing's sarcoma," Journal of Cardiovascular Magnetic Resonance, vol. 8, no. 6, pp. 789-791, 2006. 


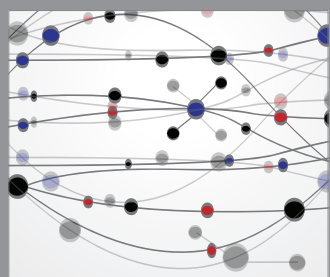

The Scientific World Journal
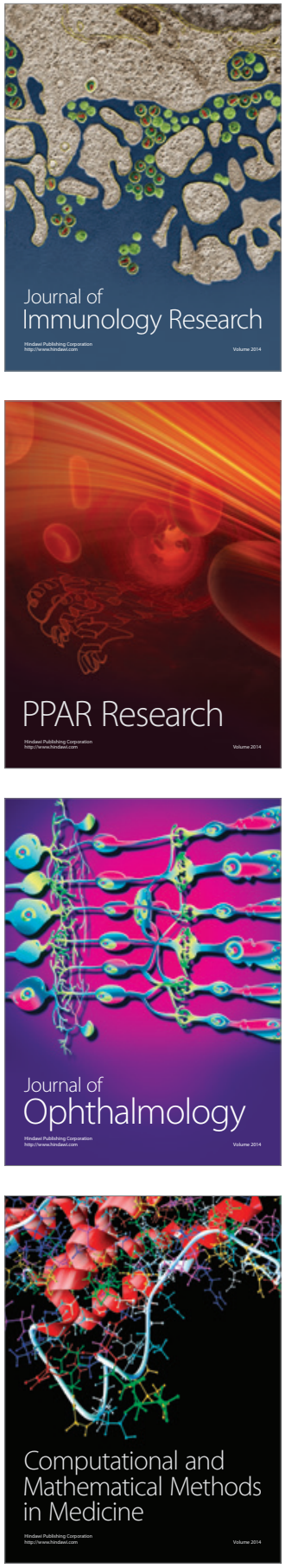

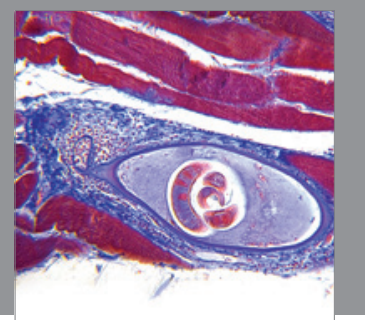

Gastroenterology

Research and Practice
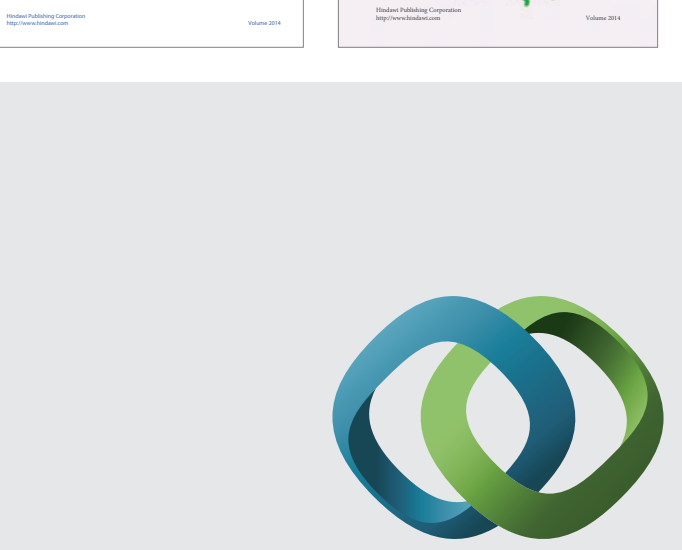

\section{Hindawi}

Submit your manuscripts at

http://www.hindawi.com
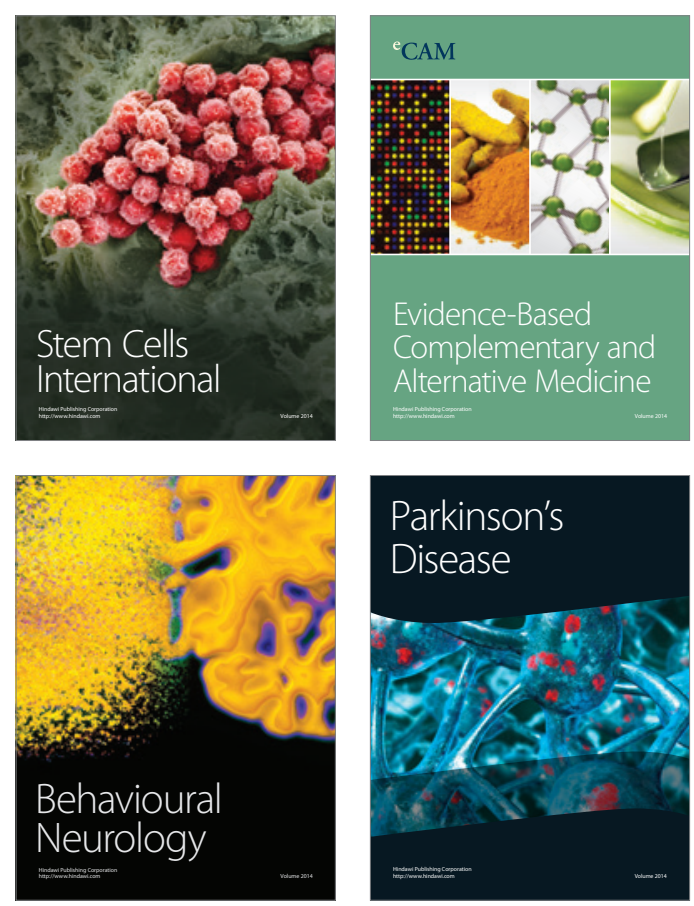

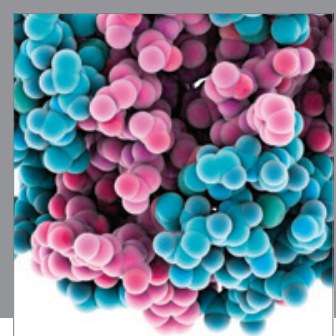

Journal of
Diabetes Research

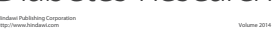

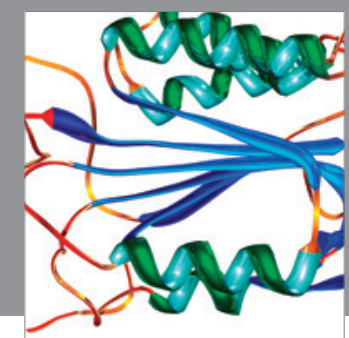

Disease Markers
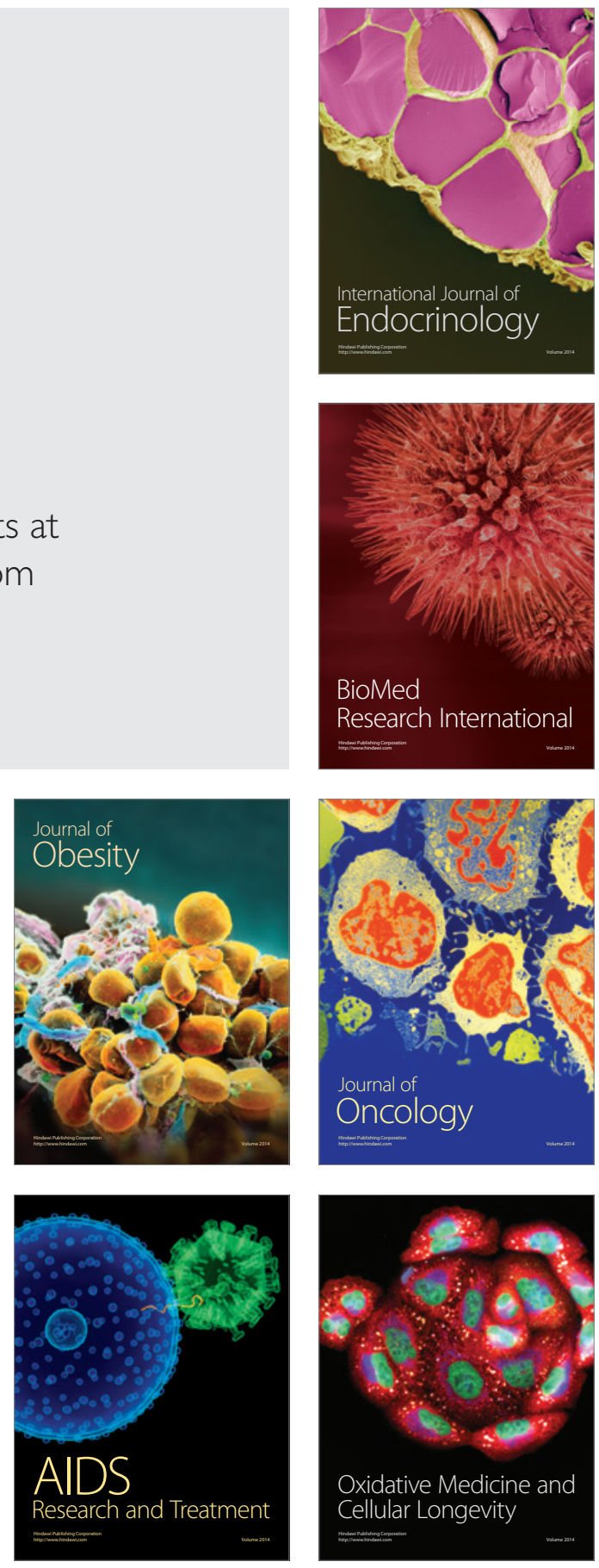